\title{
MAGNITUDE ABSOLUE MOYENNE DES RR LYRAE
}

\author{
A. $\mathrm{HECK}$ \\ Institut d'Astrophysique de l'Université de Liège, Belgique
}

\begin{abstract}
Résumé. Par une méthode basée sur le principe du maximum de vraisemblance, nous obtenons, pour un échantillon de $102 \mathrm{RR}$ Lyrae, $\bar{M}_{\mathrm{pg}}=0.6 \pm 0.2$ et $\sigma_{M_{\mathrm{pg}}}=0.5 \pm 0.2$. Les résultats de Clube et Jones (1971) ne sont pas confirmés. L'étude des corrélations entre $\bar{M}$, la période, l'indice de métallicité $\Delta S$ et l'amplitude de variation en magnitude montre que, sur la base des données actuelles, aucune corrélation significative ne peut être établie.
\end{abstract}

Abstract. By a method based on the principle of maximum likelihood, we obtained for a sample of 102 RR Lyrae stars $\bar{M}_{\mathrm{pg}}=0.6 \pm 0.2$ and $\sigma_{M_{\mathrm{pg}}}=0.5 \pm 0.2$. We could not confirm the results of Clube and Jones (1971). The study of the correlations between $\bar{M}$, the period, the abundance parameter $\Delta S$ and the light variation amplitude showed that on ground of the present data, no significant correlation can be established.

Le travail dont il est question ici consiste en l'application de la méthode basée sur le principe du maximum de vraisemblance, mise au point par Jung (1968, 1970), à un échantillon de RR Lyrae.

L'échantillon que nous avons considéré est constitué des RR Lyrae tabulées par Van Herk (1965) dont les mouvements propres et la vitesse radiale sont connus. Nous avons éliminé les étoiles à grande erreur sur les mouvements propres ( $>$ ".014) et deux étoiles (RV Cap et AS Vir) dont les vitesses spatiales sont très importantes. Il est en effet peu probable que ces étoiles appartiennent à une population parente. L'échantillon résultant se composait de 102 étoiles.

Les erreurs probables sur les mouvements propres ont été prises en considération. Le biais introduit a été calculé à l'aide d'expériences numériques qui ont en outre permis de déterminer la précision des résultats.

Les magnitudes sont des magnitudes photographiques et elles ont été corrigées de l'absorption par la loi:

$$
A_{\mathrm{pg}}=0.19[1-\exp (-0.01 r|\sin b|)] /|\sin b| .
$$

Compte tenu du biais, le résultat final est le suivant:

$$
\begin{gathered}
\bar{M}_{\mathrm{pg}}=0.6 \pm 0.2 \\
\sigma_{M_{p 8}}=0.5 \pm 0.2 .
\end{gathered}
$$

Un problème intéressant soulevé par Clube et Jones était de savoir si les mouvements propres sont affectés d'erreurs systématiques provenant d'erreurs sur le mouvement solaire, particulièrement dans la direction de la rotation galactique, des étoiles de référence. La comparaison des mouvements propres des étoiles communes à notre échantillon et à l'AGK3 nous a amenés à conclure à une erreur systématique de 0 .006. La correction des mouvements propres sur base de ce résultat ne conduit cependant qu'à des modifications négligeables.

Nous avons également appliqué la méthode du maximum de vraisemblance à 
l'échantillon utilisé par Clube et Jones (1971) en vue de confirmer ou d'infirmer leurs valeurs $\bar{M}_{v}=1.30$ et $\sigma_{\mathrm{M}_{v}}=0.5$. Mais, même en tenant compte d'une erreur systématique sur les mouvements propres $(0.015)$, nous ne pouvons pas confirmer ces résultats. Les valeurs obtenues (après correction du biais et de l'erreur systématique) sont $\bar{M}_{\mathrm{pg}}=0.6$ et $\sigma_{\mathrm{M}_{\mathrm{pg}}}=0.8$. Nous rappelons que, selon Van Herk (1965), il faut ajouter la correction $(B-V)_{o}=0.19$ aux magnitudes visuelles pour les transformer en photographiques, ce qui accroît encore le désaccord.

Enfin, nous avons recherché les relations susceptibles d'exister entre $\bar{M}$, la période, l'indice de métallicité $\Delta S$ et l'amplitude de variation en magnitude. Les résultats montrent que si certaines relations paraissent être réelles en considérant les valeurs moyennes, les grandes dispersions nous amènent plutôt à considérer de telles relations avec prudence. Il serait vivement souhaitable de reprendre ces calculs avec un échantillon plus important! Pour plus de détails, nous renvoyons à Heck (1972).

\section{Bibliographie}

Clube, S. V. M. et Jones, D. H. P.: 1971, Monthly Notices Roy. Astron. Soc. 151, 231.

Heck, A.: 1972, Astron. Astrophys. 21, 231.

Herk, G. van: 1965, Bull. Astron. Inst. Neth. 18, 71.

Jung, J.: 1968, Bull. Astron. Paris Ser. III, 3, 461.

Jung, J.: 1970, Astron. Astrophys. 4, 53. 